\title{
Some aspects on air pollution in historical, philosophical and evolutionary context
}

\author{
A. A. Berezin ${ }^{1} \&$ V. V. Gridin ${ }^{2}$ \\ ${ }^{I}$ Department of Engineering Physics, McMaster University, Canada \\ ${ }^{2}$ Department of Chemistry, Technion-Israel Inst. of Technology, Israel
}

\begin{abstract}
This paper discusses import of physical ideas on management and human response to Air Pollution (AP). It looks into some historical, evolutionary, ecological and philosophical aspects of AP. Paper emphasizes a need for more extended philosophical reflections on AP which should go beyond mere technical issues of monitoring and control.

Keywords: air pollution, biosphere, evolution, adaptation, hormesis, quantum physics, chaos, ecology, singularity theory, management of air pollution, fuzzy logic, random models.
\end{abstract}

\section{Introduction}

A standard orthodox view of Air Pollution (AP) is that it is, so to say, "by definition" an unquestionably negative happening, which - ideally - should be eliminated or, at the very least, minimized and controlled. Such a thesis, in-spite that it is almost universally accepted as an Axiom of Truth, obfuscates the fact that AP (as well as other forms of pollution) is an inevitable and, in a sense an integral, component of modern technological civilization and, perhaps, can even exhibit some redeeming aspects. In other words, upon a more critical look it should be recognized that modern AP is a direct result of technological and social development of humankind.

In this paper we look at AP within a more encompassing philosophical vista. Instead of looking at AP as a singularly negative phenomenon, we outline a more pragmatic and adaptive approach to the whole gamut of AP aspects as well as air quality in general. As an objective phenomenon, AP is a (practically inevitable) consequence of such key aspects as industrialization, transportation and 
urbanization. These are coupled with an (often excessive, wasteful and poorly regulated) use of natural resources (oil, gas, water, etc).

The authors, who are physicists, are looking at the above issues through prism of possible physical connections and analogies $[1,2]$. This is a typical way of how methods of physics (especially, theoretical physics) make their "intrusion" into other areas of technology, science and social knowledge. Numerous examples of that can be found is such diverse areas as global economy and dynamics of stock market, psychology and psychiatry, conflict resolution studies, population dynamics, ecology, and even in inter-human relationships and sexology.

This paper has a two-fold goal. One aim, in extension of our previous work $[1,2]$, is to discuss possible relevance of a variety of physical effects to AP. The other aim is to discuss import of physical mind-frame and some ideas of quantum and non-linear physics for various aspects of human response to AP. The latter include issues of regulatory and managerial nature, such as methods and strategies of decisions making regarding AP.

\section{Brief history of air pollution}

Paraphrasing title of the bestselling book by the physicist Stephen Hawking "Brief History of Time" here is, a somewhat grotesque, "brief history of air pollution" in a concise hindsight.

\subsection{Air pollution before the Industrial Age}

Apparently, every generation tends to over-rate problems it faces. The Past almost invariably bears a flavor of the "golden Age". So is the mythology that in the past the "water was clean and the air was fresh". The question here, of course, what precisely "clean" and "fresh" mean? As argued below, these definitions are not always a clear-cut matter.

In a pre-industrial age, almost all energy was produced by burning of wood, primarily for heating and cooking purposes. Early metallurgy also used coal, but due to a general scarcity of the population, these activities did not generate a significant contribution to global (and in most cases - even local) AP levels.

\subsubsection{Natural versus human-induced air pollution}

The very meaning of the word "pollution" implies that there is some departure from the norm. Hence, in order to define what is AP, one must first define the base-line, that is what is the "air without AP". Apparently, the best we can take for that is a standard chemical composition of the air. In terms of volume percentage, Earth's atmosphere is $78 \%$ nitrogen, $21 \%$ oxygen, $0.9 \%$ argon, and $0.04 \%$ carbon dioxide with very small percentages of other gases. Our atmosphere also contains water vapor, normally about $1 \%$. In addition, (natural) Earth's atmosphere contains traces of dust particles, pollen, plant grains and other solid particles. These latter additives can, in a certain sense, be referred as "natural pollution", although this term is not commonly used. 


\subsubsection{Climate oscillations}

It is an established fact that the Earth's climate constantly experiences changes at a variety of time scales. Some of these data are based on the measuring the relative concentrations of stable isotopes in ancient ice. Isotopic methodology is also a powerful investigative tool in several major areas of science and technology [3] and its potential significance for AP-related issues is still underappreciated. One such method can be the use of sensitive Doppler spectroscopic monitoring of the polluted atmosphere because thermal velocities of various isotopic species (such as, e.g., isotopically different $\mathrm{CO}_{2}$ molecules) are slightly different.

\subsubsection{Volcanic and seismic activity}

Volcanic eruptions produce local variations in air composition due to ejection of sulfur dioxide, other gases, and solid particulate. It is logically consistent to view such variations as naturally induced AP. When these eruptions are of major magnitude, changes in the atmosphere can become temporarily global. Possible examples are historical Thera eruption in Greece ca. 1650 BCE, which is believed to destroy the Minoan civilization, or Krakatau eruption of 1883. In spite that the atmosphere as a whole "cleans itself" after such eruptions in a few short years, the changes in opacity affect vegetation, which, in turn, may produce shifts in $\mathrm{CO}_{2}$ concentration. This fact even further complicates the present-day Global Warming controversy [4]. Another factor is the effect of ocean plankton which plays a key role in a $\mathrm{CO}_{2}$ balance in the atmosphere. This dynamics can be affected by major seismic activity at the ocean floor, as well as climatic changes leading to re-arrangements of ocean currents.

\subsubsection{Cosmic factors}

There are multiple factors, external to the planet, that have direct and indirect effect on the types and levels of AP on a global scale. Variations in Solar activity (Sun spots, etc), fluxes of high energy cosmic particles, density of inter-planetary dust and occasional asteroid hits (e.g., Tunguska impact of 1909) - all affect AP to a variable degree. At the same time, such aspects as possible gravitational effects on AP coming from the Moon or other planets have not been studied systematically. Since effects from the Moon on Earth are quite significant (e.g., daily tides or female monthly cycle which is believed to be period-locked with Moon's orbital motion), similar direct gravitational effects on AP are in all likelihood can also be noticeable (e.g., tide-like waves in AP patterns).

\subsection{Industrial era}

Ina context of AP the beginning of industrial era can be traced to advent of a massive use of coal and (later) an introduction of the first steam engines which later led to the steam-powered ships and railways.

While with some stretch of imagination it is possible to count the Modern Era from about 1860-1870 (e.g., Civil War in USA and Franco-Prussian War in Europe), the most common "starting date" conveniently coincides with the beginning of the 20 -th century. The first recorded air flight on a heavier-than-air 
machine (Brothers Wright, 1903) is what we usually take as the beginning of modern ("our") civilization in a proper sense.

\subsubsection{Massive industrialization}

Along with the continuing use of coal, beginning of the 20-th century saw a rapid advent of oil, which within a few decades has replaced coal as the Number One commodity in the world economy. Presently, AP due to the exhausts of billions of internal combustion engines in cars and several hundred thousands air flights every day, is the prime contributing factor to the world-wide AP. In parallel with this, there are at least three other factors contributing to AP in a significant way, namely, chemical industry, agriculture and dumping sites.

\subsubsection{Chemical industry}

While coal keeps contributing to AP (now mostly through $\mathrm{CO}_{2}$ and $\mathrm{NO}_{\mathrm{x}}$ from steel production and coal electro-power plants), chemical industry has become a major contributor too. The diversity of products of chemical industry is enormous and beyond the scope of this article. It is sufficient to mention plastics, paints, fragrances, pharmaceuticals, industrial chemicals, agricultural fertilizers and all kinds of modern food processing industries. Another (growing) outlet is the semiconductor and computer industry which produces a variety of potentially toxic waste (e.g., computer batteries, metal parts, liquid crystal panels, etc).

\subsubsection{Agriculture industry}

A separate category is the contribution of the intestine gases (mostly, methane) from zillions of agricultural animals all across the globe. While by obvious reasons this peculiar contribution may be somewhat downplayed in public perception, it, on a balance, contributes quite significantly to AP. In the same category, one can mention such an unpalatable (but inevitable) issue as numerous sewage treatment facilities, many of which are far from perfect quality in terms of purification of escaping gases and poor water treatment.

\subsubsection{Dumping sites}

Problems related to handling of ever-growing heaps of trash is taking greater and greater place among public concerns. It is becoming a hotly debated social and political issue. Municipal and regional elections are often run primarily on these concerns. Nobody wants trash and garbage around, yet we all have little choice but to produce more and more of it. Underground trash burials is only a palliative solution, firstly, because greater and greater scarcity of "free land", and, secondly, because decomposition gases tends to leak even from beneath many meters of the ground.

\subsubsection{Waste incineration}

Incineration of combustible waste goes on at various scales - from private local incinerators to major centralized incineration factories. In-spite that the exhausts of incinerators are usually equipped with a variety of filters and electrostatic precipitators, some fraction of gases (primarily, VOC - volatile organic compounds) inevitably escape to the atmosphere. 


\subsubsection{Other forms of pollution}

While AP is one of the major points of public concern (especially in some megalopolises as, e.g., Los Angeles), other forms of pollution are entering into the focus of attention as well. Among them, pollution of waters (both inland waters and oceans) and soils are the most obvious forms, but some more subtle and less recognized forms of pollution are also taking a growing part, primarily, electromagnetic and informational pollution [1]. There are several mechanisms of how these forms of pollution may interact with AP.

\section{Ecological and physical aspects of air pollution}

\subsection{Human adaptation to Air Pollution}

One of the prime features of human race and the biology at large is the idea of adaptation to the changes in the environment. This adaptation is highly interactive and includes both positive and negative feedback. It also proceeds at a variety of characteristic time scales and, hence, using the terminology of theoretical physics, can be called time scale invariant. This is one of the reasons for massive use of ideas of chaos and nonlinear dynamics in the present-day ecological and evolutionary studies (e.g., [5]). In this context AP is not an exception, and human adaptation to it (as well as its practically possible control) is an on-going reality which should be analyzed and accepted.

\subsubsection{Evolutionary (Darwinian) adjustments}

According to the standard Darwinian concept, the two prime aspects of biological evolution are adjustment to the (changing) environments and survival of the fittest. At this moment, there is no singular explanation of what exactly drives the evolution. Apart from theological and mystical explanations, the most scientifically sound line of arguing goes along theory of spontaneous pattern formation and complexity emergence. These ideas are taken from physics, especially from Dynamics of Non-Linear Systems, which include such versatile analytical tools as the Theory of Chaos, Theory of Catastrophes and the notion of Strange Attractors. The latter illustrates the idea that the (evolving) system has some "goal" which it is striving to reach ("attracted to").

\subsubsection{Hormesis effect (chemical, radioactive, electromagnetic)}

Hormesis effect is an umbrella term for a non-linear dose response of organisms to various (defined as harmful) environmental factors. It is a part of social trivia that harmfulness or usefulness of various factors often depends on the dose. Many heavy metals (manganese, vanadium, chromium, etc) are poisonous, yet some of them are included in poly-vitamin pills in micro doses.

In a proper sense, hormesis is a dose-related, allegedly beneficial, response to a number of (nominally harmful) environmental factors. Examples are chemical, radioactive, and electromagnetic hormesis. It is established that small doses of radioactivity can stimulate human immune system and lead to a better adaptability and strengthening. At the same time, it is known that natural 
radioactivity is one of the prime factors leading to genetic mutations (atomic scale changes in the genetic code) and this, in turn, fosters evolutionary changes. Likewise, because AP in general has many chemically diverse contributors, it is quite likely that some of them in low doses may be more beneficial than harmful. People living in large cities usually adapt to moderate levels of AP. At the same time, when people permanently living in remote areas with a pristine environment and clean air suddenly come to large cities with their smogs and car exhausts, they often experience adverse health reactions. While this is in no way an advocacy for more AP, in view of these authors the role of AP as adaptability factor should be recognized and studied in a more focused way.

\subsubsection{Societal reflection and controversies}

For all practical purposes no form of pollution can be completely eliminated. Therefore, excessive demands of "zero-tolerance" to AP are untenable. Likewise, the ideas of Deep Ecology and sustainability ("back to nature" movements) are, for the most part, naive and illusive. At the same time, because these views look straightforward and appeal to large segments of the population, they are becoming a part of a broad environmental discourse and generate within them some good ideas. Some spin-off include growing activity in a search for alternatives to fossil fuels (e.g., electric cars) and alternative forms of power generation (solar, wind, ocean tides, etc) or more environmentally friendly goods, like small wearable electronics, etc.

\subsection{Critical effects in air pollution}

In physical terms AP is a distributed phenomenon which most commonly treated as a (quasi-continuous) diffusion process. It is known, both phenomenologically and theoretically, that quasi-continuous dynamics often leads to singular manifestations with a highly non-uniform concentrations of energy and particles. An example is a soliton wave which propagates as a localized crest. Similar effects of self-induced coagulation of particulate in AP may happen either spontaneously or be triggered by outside factors, as, for example, coherent electromagnetic waves [1]. Some more subtle effects as gravitational reduction of the wave functions of micro-particulate and aerosols [2] may further contribute to coagulation dynamics.

\subsubsection{Bifurcations and Chaos Theory}

Chaos Theory is presently a significant segment of physics with applications to many diverse areas ranging from climatology to economics. One of its key ideas is utter sensitivity to the initial conditions which is a characteristic feature of many non-linear systems. This is the so called "Butterfly Effect" which a code name for a fast (exponential) divergence of the phase trajectories in a phase space of the system. This feature often manifest itself in AP, for example a slight change in a wind pattern can drastically affect the deposition of hard particulate and air quality at a particular locality.

The said Butterfly Effect has two consequences with opposite implications to the human response to AP and its management. On one side, it drastically lowers 
the level of possible predictability of AP dynamics, especially on longer time scales (say, many days). On the other side, high sensitivity to the initial conditions allows very small perturbations to affect the dynamics in a macroscopic way. This situation is commonly known in physics when very weak forces radically change the outcome of the process. An example of the latter is a beta-decay of some isotopes. In case of AP this, potentially, leads to a possibility to affect patterns of AP propagation by energetically small (but informationally structured) pulses [1]. One example can be application of Laser pulses similar to the methodology of Laser Doppler cooling. This could selectively affects various categories of pollutants due to differences in their spectroscopic properties and dispersion in their thermal velocities.

\subsubsection{Theory of Catastrophes}

Theory of Catastrophes (Rene Thom) is one of the precursors of the modern Chaos Theory. Both have ideas of bifurcation (forking paths) and both are applicable to systems with jump-wise transitions between dynamical regimes. In case of AP, the Theory of Catastrophes can describe sharp transitions in AP descriptors (pollution level and pollution content in time and spatial variations).

A historical example is the so called Toba Catastrophe - a eruptions of a super-volcano some 70,000 years ago. It lead to a catastrophic increase of AP and a drop of a global temperature by several centigrade. Analysis of human genetic code indicates that the proto-human population dropped to a few thousand breeding pairs worldwide. As a result, this was a likely bottleneck happening in the emergence of modern humans. Thus, one can, somewhat metaphorically, say that the origin of (modern) human species is a direct result of a sharp singular increase of AP level for a short time interval.

Another finding of Bifurcation Theory and Chaos is the existence of windows of Order within Chaos. In terms of AP patterns and dynamics this can manifest itself in the formation of AP clusters showing some spatial regularity across the Globe, in some analogy with the known electrostatic problem of the arrangement of $\mathrm{N}$ charges over the surface of a sphere.

\subsubsection{Ergodicity Principle and dynamical singularities}

Ergodicity is a term which originated in statistical physics and thermodynamics (e.g., Boltzmann's "H-theorem") which posits that a dynamical system with macroscopic number of degrees of freedom (e.g., a mole of a gas), given a sufficient time, will pass through all microscopic states in its phase space. As a complimentary term to the notion of entropy, Ergodicity Principle demonstrates the existence of fluctuations of any scale and shape. Examples of the latter are the formation of extremely dense pockets of AP in some localities (e.g., notorious smogs in Los Angeles area), which may be in excess of what actual emissions entail. In terms of the Chaos Theory, these dynamical regimes (quasi singularities) can be described as Strange Attractors (sets of points in the phase space to which system periodically returns). 


\subsection{Air pollution in evolving biosphere}

Within a view of the human race as a part of the evolving biosphere, AP which is due to human activity, should be considered as an organic part of the biosphere in whole. As such, AP (both natural and human made) is neither "bad" nor "good", but should be treated in pragmatic terms. The latter requires balancing of monitoring with technologically and economically available control and adaptability.

\subsubsection{Self-regulating systems}

The above described Ergodicity Principle stays in some dialectical opposition to the Second Law of Thermodynamics (law of entropy increase), because it points to an inherent ability of non-linear systems to self-organization and complexity emergence. This ability of self-regulation is thought to belong to the biosphere at large ("Gaia" hypothesis), as well as to separate and adjacent components of it, of which Earth's atmosphere (and hence, AP) is a part.

\subsubsection{Gaia Hypothesis}

Within the context of Gaia Hypothesis (James Lovelock), atmosphere is not just an outside non-organic (non-living) entity in which we happen to be embedded, but an immanent part of the biosphere itself. This idea can be seen as an extension of Le Chatelier-Braun principle to the entire global system comprising of biosphere and atmosphere. For that matter, a relatively small (in terms of bulk concentration) additives to the air which are brought by AP may also play their role in Gaia, similarly to microelements in human nutrition.

\subsubsection{Air pollution in toxicity context}

At the present moment there is no sufficiently developed understanding of the effects of toxicity of chemical elements and compounds versus the adaptational effects of hormesis (Section 3.1.2). In a crude sense, the first are seen as "bad" while the latter (hormesis) as "good" (boosts the immune system). However, the juxtaposition of these two is more subtle, and proper understanding of mutual interactions in this tandem may call for the application of some ideas of theoretical physics.

One of the perils (not yet properly appreciated) of the modern technological age lies in the use of ultra-pure materials. Currently available technological methods allow production of materials purified to a degree several orders of magnitude higher than can be found in nature. For example, semiconductor industry uses such ultra-pure materials as GaAs or CdTe. All these elements are poisonous to human. And yet, trace amounts of them are all around and we all take them to our bodies through the nutrition and inhaling. So, where is the catch here?

In physics there are concepts of "dressed particles" and quantum states. This means that the particles (e.g., impurity atoms) surrounded by other particles are screened in their interactions with other parts of the system. Examples of these dressing (screening) effects are Cooper pairs in superconductivity, or polarons in 
crystals. Polaron is a "dressed" electron and its "dress" is a cloud of phonons (lattice vibrations).

Likewise, toxic elements in there natural (not pure) forms are surrounded by other compounds. This mitigates and passivates their toxicity action. In fact, in non-pure form their toxicity can even turn into a health benefit - many elements toxic in pure form (selenium, manganese, vanadium) are part of poly-vitamin supplements. Effects of super-pure materials (which do not have the benefit of this screening effect) are not well known, but a reasonable assumption is that their toxicity action can be enhanced by many orders of magnitude. It is well known that drinking exclusively distilled water is harmful. This is because distilled water is deprived of microelements and trace additives essential for our physiology. The latter are contained in natural water (even filtered). Similarly, a strive to an ultra pure air can, in fact, be more hazardous than beneficial. In other words, some level of AP (and chemical diversity brought by AP) should not only be tolerated, but should be an immanent part of our existence.

\section{Future scenarios and human response}

In this article we adopt the view that AP is an inherent part of the anthropogeneic ecology. This vista suggests a few possible future scenarios for the role of AP in a social fabric, as well as to the managerial response to it.

\subsection{Self-Organized Criticality}

Idea of SOC (Self-Organized Criticality) lies within modern Theory of Chaos [1] and points towards the formation of high level instabilities in non-linear systems. In a nutshell, SOC model accounts for a gradual build-up of instability in a nonequilibrium system and its subsequent discharge in a form of sudden avalanches. These avalanches can happen at a variety of spatial-temporal scales (scale invariance) and proceed in a stochastic non-coherent form (e.g., luminescence), or in a form of highly correlated singular discharge (e.g., coherent laser emission). A popular visual model for SOC is a collapse of a sand pile with a gradual adding of more sand. As such, AP is a complex heterogeneous system having gaseous components (nitrogen oxides, sulfur dioxide, VOC, etc), liquid aerosols and solid components (hard particulate). Therefore, dynamical modifications of AP patterns can happen at a broad range of scales including sudden singular-type phase transformations. This makes AP another test model for the application of SOC methodology.

Above mentioned (Section 3.2.1) triggering of macroscopic phase changes in AP by targeted laser pulses can be further extended to include upper layers of the atmosphere, in particular, ionosphere. At the moment, there is no comprehensive understanding of the links between processes in the ionosphere and groundbased AP. However, due to high responsivity of ionosphere to outside factors (example is Aurora Borealis) and the fact that is carries a distributed electrostatic charge, all effects of electrostatic phase transitions [2] and self-organization in charged plasma can be potentially induced by properly designed perturbations 
(e.g., laser beams). Redistribution of charge density in ionosphere can, in turn, reflect on AP at the ground level.

\subsection{Singularity Theory}

The ideas of an impending Singularity in technological, ecological and demographic fabric of the global society are getting a growing factual support [6]. From global economic competition and radical shifts in international markets to such technological trends as expected breakdown of the Moore's Law (doubling of computer chip power every 18 months) and emergence of Quantum Computing [7], one can foresee a radical (singular) shift. From local ecological patterns we come to a global ecological linkage of which AP is an inevitable component.

A corollary to the Singularity Theory are the package of ideas known as Trans-humanism - a transcendence of human realm into a post-human existence. The suggested scenarios here run from the apocalyptic pictures of global ecological collapse (to which AP will likely to contribute) to the visions of the ascendance of a New Golden Age when all acute problems of today are harmoniously solved. While either of these extremes is unlikely to come in a pure form, the suggested time-frames for them are surprisingly short (30 to 50 years hence).

\subsection{Decision making in air pollution management}

Managerial decisions concerning AP require balancing of numerous (often conflicting) objectives and vested interests (technological, social, economical, political, developmental, health-related, etc). Additionally, scientific (and even philosophical) aspects of AP issues are often complicated and controversial, with different (often utterly adversarial) viewpoints (e.g., Human-made Global Warming controversy). Below we suggest a few thoughts on these issues.

\subsubsection{Fuzzy Logic}

Fuzzy Logic (FL) nowadays is an accepted mainstream technique for the choosing a strategy in multiple-choice situations. In fact, numerous automata (like washing machines or photocopiers) have often build-in (embedded) FL microprocessors to select performance regimes.

\subsubsection{Random strategies}

Decision making by applying random strategies has a long history. Sometime, they are preferable to the "first choice" (most optimal) solutions. Dodges in Venice and judges in some jurisdiction were selected by a draw. Some earlier military manuals suggested random choice (e.g., by dice) of strategic moves (e.g., selecting mounting passes for the army). Such a strategy makes more difficult for the adversarial commander to guess the most likely route which would likely be taken on the basis of rational optimization. Randomness (dicing) removes such rational optimization and at best the adversary can do the same to do his own dicing which renders his guessing purely probabilistic. Likewise, 
qualitative and quantitative decisions on AP management, may include random choice (perhaps, with some weighting factors) among several educated guesses.

\subsubsection{Adoption of second and third-best options}

Adaptational Modelling presumes an outlining of managerial decisions through highly interactive (non-linear) linkage to a dynamically changing situation. As such, AP exhibits a volatile, ever-changing, spatial-temporal pattern. This makes it prone to the application of the ideas of Strange Attractors of the Chaos Theory. In this context, an optimal (or quasi-optimal) managerial solution aimed on a particular (local) aspect of AP is envisioned as a point on a phase trajectory of a (multidimensional) space of possible solutions. This is a generalization of the decision making based on FL for the case when the number of parameters are much greater that the traditionally used in FL methods. In this vein, a correction (and possible improvement) of the standard FL method may be to choose the second (or third, etc) option as the actually accepted managerial decision.

Prime problem with most environmental problems, AP including, that there management requires a simultaneous taking into account of numerous, often contradictory, factors and interests. In the spirit of emergent thinking along the ideas coming from physics (such as superposition principle, mixed quantum states, quantum non-localities, classical and quantum chaos, least action principles, etc), the viable strategy may be sought along the holistic approach which takes a singular view of a situation as a whole, rather than weighting separate parts of the scenario. This is applicable to AP problems as well as a variety of environmental and ecological issues at large.

\section{Conclusion}

This article present AP as a multi-sided challenge which, far from being uniformly "negative", may have some redeeming aspects (e.g., hormesis and ecological and genetic adaptation). Therefore, analysis of its structure and implications, as well as managerial response to it, require interdisciplinary approach, some aspects of which are outlined above. Ideas drawn from the work of such visionary scientists as Roy Kurzweil [6], Roger Penrose [8], James Lovelock [9], Rupert Sheldrake [10], or Seth Lloyd [11] (to name a few) can serve as a fertile ground for further contemplations of local and global solutions. In this regard it appears especially promising to explore the linkages of AP with other fundamental planetary forces and interactions (gravitational, electromagnetic, and quantum). This can further contribute to the understanding of key aspects of AP as well as foster the development of methods of its monitoring and control.

In particular, Lloyd [11] entertains the premise that the dynamics of large systems can, in fact, resemble the operation of quantum computers [7]. In-spite that such an idea may appear far fetched, it is gaining observational and theoretical support. Its application to the Earth's atmosphere with its AP as a sort of embedded neural network (interactive particulates and aerosols), can turn out 
to be relevant for the deeper comprehension of processes of chemical selfregulation in the Earth's atmosphere.

\section{References}

[1] Berezin, A.A. \& Gridin, V.V., Electromagnetic and informational pollution as a co-challenge to air pollution. Air Pollution XVI, ed. C.A. Brebbia \& J.W.S. Longhurst, WIT Press: Southampton, Boston, pp. 533542, 2008.

[2] Berezin, A.A., Quantum effects in electrostatic precipitation of aerosol and dust particles. Air Pollution XIII, ed. C.A. Brebbia, WIT Press: Southampton, Boston, pp. 509-518, 2005.

[3] Berezin, A.A., Isotopicity: Implications and Applications, Interdisciplinary Science Reviews, 17 (1), 74-80, 1992.

[4] Lomborg, B., The Skeptical Environmentalist. Cambridge University Press, 2001.

[5] Kauffman, S.A., The Origins of Order - Self-Organization and Selection in Evolution. Oxford University Press, New York/Oxford, 1993.

[6] Kurzweil, R., The Singularity is Near (when humans transcend biology. Penguin Books, New York, 2005.

[7] Berezin, A.A., Quantum computing and security of information systems, Safety and Security Engineering II, eds. M. Guarascio, C.A. Brebbia, F. Garcia, WIT Press: Southampton, Boston, pp. 149-159, 2007.

[8] Penrose, R., The Road to Reality: A Complete Guide to the Laws of the Universe. Jonathan Cape, London, 2004.

[9] Lovelock, J., The Revenge of Gaia: Why the Earth is Fighting Back - and How We Can Still Save Humanity. Allen Lane, Santa Barbara (California), 2006.

[10] Sheldrake, R., The Presence of the Past: Morphic Resonance and the Habits of Nature. Times Books (Random House), New York, 1988.

[11] Lloyd, S., Programming the Universe: Is the Universe actually a Giant Quantum Computer? Vintage Books (Random House), New York, 2007. 